\title{
Regulation of adrenocorticotropic hormone secretion: lessons from mice deficient in corticotropin-releasing hormone
}

\author{
Audrey Seasholtz \\ University of Michigan, Mental Health Research Institute, 205 Zina Pitcher Place, \\ Ann Arbor, Michigan 48109-0720, USA. \\ Phone: (734) 936-2072; Fax: (734) 647-4130; E-mail: aseashol@umich.edu.
}

"Stress is life and life is stress." The words of Hans Selye from the 1940s still ring true today. Selye recognized that physical, emotional, and environmental challenges (stressors) elicit a variety of physiological responses and that our ability to respond and adapt to these stressors is critical to our survival. In the 50 years since these initial observations, we have learned a great deal about the complex system that allows us to maintain homeostasis in the resting state and to respond appropriately to stressors, yet much remains to be learned about the regulation of this system.

A key component of this stress system is the hypothalamic-pituitary-adrenal (HPA) axis (Figure 1). In response to a stressful stimulus, neural inputs from the central and peripheral nervous system converge on a small nucleus in the hypothalamus (the paraventricular nucleus, or PVN) and signal for increased synthesis and release of corticotropinreleasing hormone $(\mathrm{CRH}$, also known as CRF). This 41 -amino acid peptide is released into the hypophyseal portal blood and carried to the anterior pituitary, where it binds to $\mathrm{CRH}$ receptors on the corticotropes. As with other $\mathrm{G}_{\mathrm{s}}$ protein-coupled membrane receptors, $\mathrm{CRH}$ receptors stimulate production of the intracellular second messenger cAMP. Activation of this pathway results in increased production of proopiomelanocortin (POMC) and increased release of adrenocorticotropic hormone (ACTH) and $\beta$-endorphin, bioactive proteolytic products of POMC. While CRH is widely regarded as the major hypothalamic releasing factor for ACTH, other hypothalamic compounds such as vasopressin, oxytocin, and norepinephrine can also stimulate ACTH release (at much lower potencies) or potentiate CRH-induced ACTH secretion (reviewed in ref. 1). ACTH is carried via the blood to its target organ, the adrenal cortex, where it stimulates secretion of glucocorticoids (corticosterone in rodents or cortisol in humans). Glucocorticoids then mediate a variety of metabolic effects that help the body to respond to the stressor; for example, they stimulate protein catabolism and gluconeogenesis while inhibiting peripheral glucose uptake.

Glucocorticoids also play a critical role in turning off the stress signal to maintain homeostasis in the HPA axis.

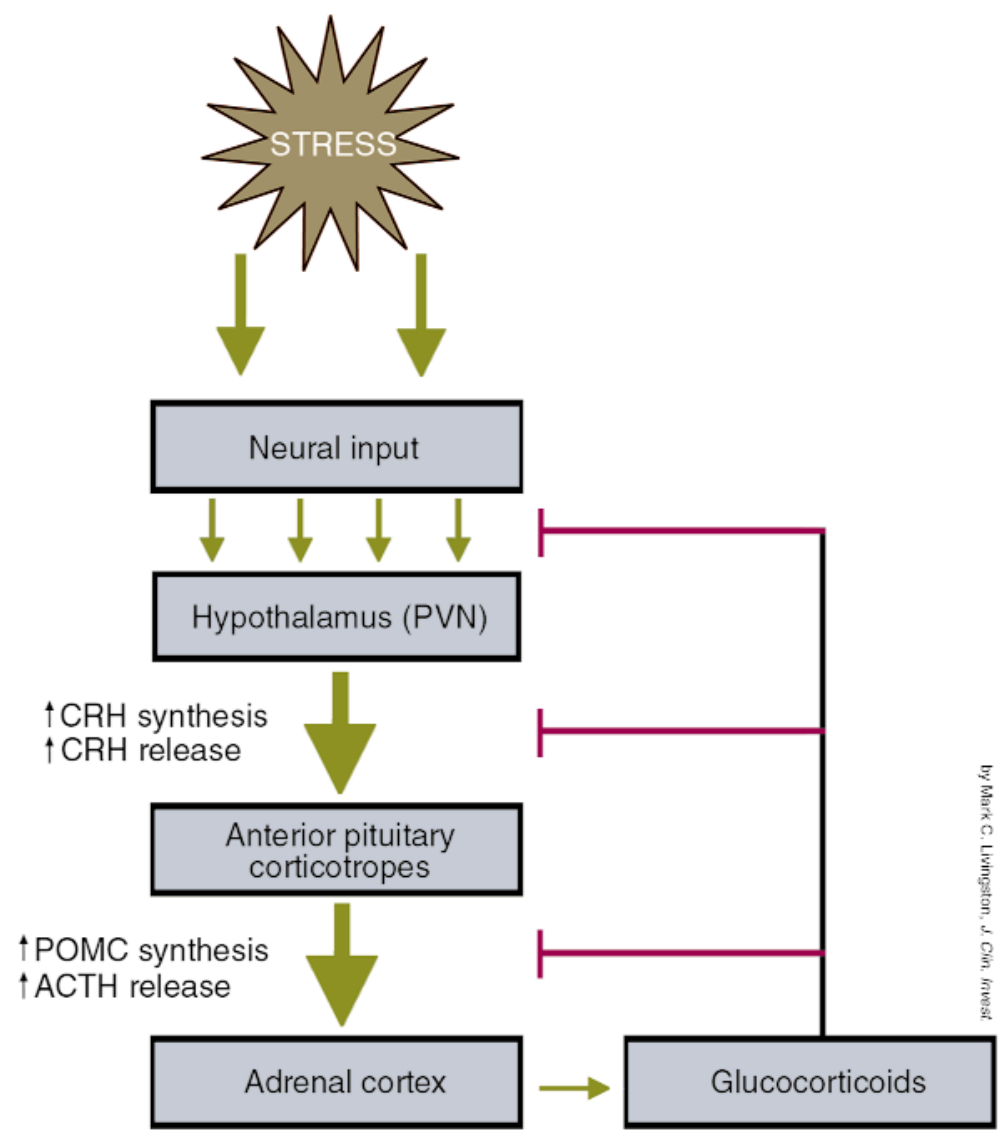

Figure 1

Schematic representation of the hypothalamic-pituitary-adrenal (HPA) axis. The initial changes in the axis in response to a stressful stimulus are shown in red. Stress increases $\mathrm{CRH}$ synthesis and release from neurons in the paraventricular nucleus (PVN) of the hypothalamus. $\mathrm{CRH}$ is carried to the anterior pituitary, where it binds to $\mathrm{CRH}$ receptors on corticotropes, resulting in increased synthesis of POMC and release of ACTH. ACTH, in turn, stimulates production of glucocorticoids from the adrenal cortex. Glucocorticoids signal for a variety of metabolic changes that allow the body to respond to the stressor. They also provide negative feedback (shown in red) at numerous levels in the HPA axis to terminate the stress response and maintain homeostasis. 
ACTH, and glucocorticoid release but then to return the system to its basal unstimulated state to maintain homeostasis. Altered HPA activation or impaired negative feedback regulation by glucocorticoids is associated with a variety of clinical endocrine and psychiatric disorders, including Cushing's disease, depression, anxiety disorders, and anorexia. A clear understanding of the regulatory mechanisms in this axis is critical to our ability to develop new treatments for these disorders.

It is not surprising, then, that changes in the glucocorticoid status of an animal result in altered POMC synthesis and ACTH release. Yet the individual contributions of hypothalamic CRH or peripheral glucocorticoids (at various concentrations) to these pituitary corticotrope changes remain controversial. Many studies have examined this question using physiological alterations such as adrenalectomy in the presence or absence of corticosterone replacement, pharmacological treatments, or neuroanatomical lesions (reviewed in ref. 2). An alternative approach, adopted by Muglia et al. in this issue of the JCI (3), involves the use of genetically altered mice - in this case, a CRH-deficient mouse.

The CRH-deficient mouse was created in 1995 by Muglia and colleagues using homologous recombination (4). These mice are completely CRH-defi- cient and exhibit chronic glucocorticoid insufficiency. Not surprisingly, they also have a dramatically impaired stress response. Interestingly, however, plasma ACTH levels in these mice appear to be normal despite the absence of CRH and altered glucocorticoid levels. Now, Muglia and colleagues have used this interesting mouse model to address the individual roles of glucocorticoids and/or CRH in POMC synthesis and ACTH release (3). Their results suggest that in $\mathrm{CRH}$-deficient mice, alterations in peripheral glucocorticoid levels can regulate POMC synthesis and ACTH production, but that changes in ACTH release in response to altered glucocorticoid levels require the presence of CRH.

While we often associate increased synthesis with increased secretion, these events are clearly distinct. Numerous studies in anterior pituitary cultures have demonstrated decreased POMC transcription by glucocorticoids and increased POMC transcription by $\mathrm{CRH}$ (5). The identification and characterization of the genomic sequences and transcription factors mediating these changes in POMC transcription remain areas of intense study. Anterior pituitary cell cultures also show calciumdependent increases in ACTH secretion with CRH treatment or increased levels of intracellular cAMP. In contrast, glu- cocorticoids alone have little or no effect on basal ACTH secretion but decrease CRH- or cAMP-stimulated ACTH secretion $(5,6)$. These in vitro results are consistent with the in vivo data presented from the $\mathrm{CRH}$-deficient mice. Additional studies on the regulation of ACTH secretion may elucidate the key roles of $\mathrm{CRH}$ and elevated cAMP levels in calcium-mediated ACTH release and identify the molecular mechanisms by which glucocorticoids inhibit ACTH secretion. These results may have important implications in the treatment of patients following chronic glucocorticoid administration or deficiency.

1. Owens, M.J., and Nemeroff, C.B. 1991. Physiology and pharmacology of corticotropin-releasing factor. Pharmacol. Rev. 43:425-473.

2. Dallman, M.F., et al. 1987. Regulation of ACTH secretion: variations on a theme of B. Recent Prog. Horm. Res. 43:113-173.

3. Muglia, L.J., et al. 2000. Corticotropin-releasing hormone links pituitary adrenocorticotropin gene expression and release during adrenal insuf ficiency. J. Clin. Invest. 105:1269-1277.

4. Muglia, L.J., Jacobsen, L., Dikkes, P., and Majzoub, J. 1995. Corticotropin-releasing hormone deficiency reveals major fetal but not adult glucocorticoid need. Nature. 373:427-432.

5. Gagner, J.P., and Drouin, J. 1987. Tissue-specific regulation of pituitary proopiomelanocortin gene transcription by CRH, cAMP, and glucocorticoids. Mol. Endocrinol. 1:677-682.

6. Shipston, M.J. 1995. Mechanism(s) of early glucocorticoid inhibition of ACTH secretion from anterior pituitary corticotropes. Trends Endocrinol. Metab. 6:261-266. 